\title{
La ocupación en la Gerontología: una perspectiva crítica del Modelo de Ocupación Humana
}

\author{
Occupation in Gerontology: \\ A critical perspective of the Model of Human Occupation \\ Ocupação em Gerontologia: \\ uma perspectiva crítica do Modelo de Ocupação Humana
}

\author{
Deisy Krzemien ${ }^{1}$ \\ Claudia Miranda² \\ Noelia Giselle Martín ${ }^{3}$
}

\section{RESUMEN}

Desde la Terapia Ocupacional en el campo de la Gerontología, se propone una reflexión crítica sobre el uso del Modelo de Ocupación Humana en el abordaje de la ocupación en el envejecimiento, reconociendo la emergencia de la perspectiva crítica, los aportes del paradigma del curso de vida en la comprensión holística y contextual de la vejez, y la concepción de envejecimiento saludable. Se revisan los postulados principales del modelo en cuanto a la adaptación ocupacional, la volición, la habituación, la capacidad de desempeño y los entornos en la vejez. Se analiza la participación social en actividades significativas

\footnotetext{
${ }^{1}$ Licenciada en Psicología. Especialista en Docencia Universitaria. Doctora en Psicologia. Docente, Universidad Nacional de Mar del Plata. Mar del Plata, Argentina. deisykrzemien@gmail.com (iD) https://orcid.org/0000-0003-4862-5168

${ }^{2}$ Licenciada en Terapia Ocupacional. Magíster en Psicologia Social. Docente, Universidad Nacional de Mar del Plata. Mar del Plata, Argentina. claudiamiranda2811@gmail.com iD https://orcid.org/0000$\underline{0002-3769-0426}$
}

${ }^{3}$ Estudiante, Licenciatura en Terapia Ocupacional, Universidad Nacional de Mar del Plata. Mar del Plata, Argentina. noeliagisellemartin@gmail.com iD https://orcid.org/0000-0003-0785-9777 
de la persona mayor en la construcción de la vida ocupacional y el rol de la narrativa en la adaptación ocupacional en el envejecimiento. Esta reflexión tiene implicaciones en la fundamentación de la intervención ocupacional en el envejecimiento, tanto desde la perspectiva terapéutica como en la promoción de la salud y la calidad de vida de las personas mayores. En definitiva, el análisis favorece el uso del Modelo de Ocupación Humana en la práctica profesional gerontológica.

\title{
PALABRAS CLAVE
}

Terapia Ocupacional, gerontología, envejecimiento, personas mayores

\begin{abstract}
A critical reflection of the Human Occupation Model is proposed to address the issue of occupation in aging, in the field of Occupational Therapy in Gerontology. It recognizes the emergence of a critical perspective, the contributions of vital course paradigm to the holistic and contextual understanding of old age, and the conception of healthy aging. The model's main postulates are reviewed in terms of occupational adaptation, volition, habituation, performance capacity and environments in old age. This reflection has implications for the fundamentals of occupational intervention in aging, both from a therapeutic perspective and the promotion of health and life quality in the elderly population. In conclusion, the analysis favors the use of the Human Occupation Model in gerontological professional practice.
\end{abstract}

\section{KEY WORDS}

Occupational Therapy, geriatrics, aging, elderly

\section{RESUMO}

A partir da Terapia Ocupacional no campo da Gerontologia, propõe-se uma reflexão crítica sobre a utilização do Modelo de Ocupação Humana na abordagem da ocupação no envelhecimento. Reconhece-se a emergência da perspectiva crítica, as contribuições do paradigma do curso de vida na compreensão holística e contextual do envelhecimento, bem como a concepção de envelhecimento saudável. Os principais postulados do modelo são revistos em termos de adaptação ocupacional, volição, habituação, capacidade de desempenho e ambientes na velhice. Analisa-se a participação social em atividades significativas dos idosos na construção da vida ocupacional, assim como o papel da narrativa na adaptação ocupacional do envelhecimento. Esta reflexão tem implicações na fundamentação da intervenção ocupacional no envelhecimento, tanto do ponto de vista terapêutico, como na promoção da saúde e qualidade de vida dos idosos. Definitivamente, a análise favorece a utilização do Modelo de Ocupação Humana na prática profissional gerontológica.

\section{PALAVRAS-CHAVE}

Terapia Ocupacional, gerontologia, envelhecimento, idosos 


\section{Introducción}

El envejecimiento, como proceso de desarrollo humano, implica cambios multidimensionales y multidireccionales durante el curso de la vida (Baltes et al., 2006; Liberalesso, 2007). La vejez es un proceso dinámico y diverso, tanto en los aspectos que involucra (neurobiológico, morfológico, anatómico, psicológico, comportamental, social) y las diferencias de género, clase y cultura, como en sus cambios en el tiempo (Krzemien, 2013). En este sentido, el paradigma del curso vital (Baltes et al., 2006) permite una comprensión holística y metateórica de la naturaleza del desarrollo humano durante el curso de vida, donde cobra importancia el estudio del envejecimiento como proceso de diferenciación progresiva. En lugar de pensarse como opuestos, el desarrollo y el envejecimiento constituyen un mismo proceso de cambios que reúne influencias biológicas y contextuales, dando cuenta de una continuidad, pero también discontinuidad a lo largo de la vida (Lombardo y Krzemien, 2008).

Así pues, esta reflexión busca ampliar la teorización en torno al envejecimiento desde el campo de la Terapia Ocupacional en Gerontología, a partir del Modelo de Ocupación Humana (Kielhofner, 2011). Resulta relevante reflexionar sobre este modelo en función de los avances de sus planteamientos conceptuales respecto a la inclusión de factores sociopolíticos y económicos que, con el desarrollo de la perspectiva sociocomuntaria de la Terapia Ocupacional en Latinoamérica en las últimas décadas, permitirían un análisis más comprehensivo del envejecimiento. Con este propósito, se revisan brevemente los cambios recientes en las concepciones epistemológicas y teóricas de la Terapia Ocupacional que se derivan de la influencia de las variables contextuales y sociales, y de la perspectiva de los derechos humanos en la región; luego, se analizan los postulados principales del modelo en cuanto a la adaptación ocupacional, la volición, la habituación, la capacidad de desempeño y los entornos en la vejez, así como la participación social en actividades significativas de la persona mayor en la construcción de la vida ocupacional y el rol de la narrativa en la adaptación ocupacional en el envejecimiento.

Esta reflexión nace del interés en la renovación paradigmática que ha operado en las últimas décadas en las ciencias de la salud y en las ciencias humanas, en torno a dos cuestiones principales: primero, la preocupación por la reflexión teórica y metateórica en Terapia Ocupacional, a partir del giro epistemológico y filosófico en dichos campos del conocimiento (por ejemplo, la inclusión del paradigma de la complejidad) y su relación con los cambios culturales y sociopolíticos actuales (Gallheigo, 2012; Guajardo, 2012); segundo, la necesidad de repensar las problemáticas reales y complejas de lo cotidiano que atiende la Terapia Ocupacional en el contexto latinoamericano (Carvalho, 2018). Es preciso tener en cuenta estas consideraciones en el estudio de la vejez y el envejecimiento, con la posibilidad de repensar el enfoque conceptual, la modalidad de abordaje y la aplicación del Modelo de Ocupación Humana en el colectivo de las personas mayores. 


\section{Enfoques recientes en la literatura}

Tradicionalmente, la mayoría de los estudios, prácticas e investigaciones de Terapia Ocupacional en el área gerontológica se han centrado en aspectos patológicos asociados al avance de la edad, como el deterioro cognitivo (Fallahpour et al., 2015), el deterioro funcional (Corregidor, 2010) y las discapacidades y vulnerabilidades (Perlmutter et al., 2010). Así mismo, los estudios desde el Modelo de Ocupación Humana suelen abordar las patologías en la vejez (Oakley, 2008). De esta manera, se observan en la literatura científica investigaciones referidas a la experiencia de participación ocupacional de personas adultas mayores con discapacidades, en las cuales se restringe la consideración de las condiciones sociales y culturales en la explicación de la salud y la adaptación (Yerxa, 1990, 1998; Gallheigo, 2012; Cabral et al., 2018; Carvalho, 2018; Carvalho et al., 2018).

No obstante, en los últimos años, al tiempo que se producen avances en materia de teoría e intervención en Terapia Ocupacional en Geriatría y Gerontología, algunas investigaciones abordan cuestiones relativas a la ocupación y la salud en personas mayores sin patología ni dependencia psicofísica (Hwang, 2012; Mulholland y Jackson, 2018; Sanders, 2018). Uno de los estudios que a fines del siglo pasado inauguró el trabajo en esta línea es el titulado Occupation in Lifestyle Redesign: The Well Elderly Study Occupational Therapy Program (Jackson et al., 1998), el cual propuso una intervención preventiva de Terapia Ocupacional para el rediseño de rutinas en adultos mayores.
Así mismo, desde los años ochenta aparecen trabajos sobre envejecimiento saludable que utilizan el Modelo de Ocupación Humana (Elliott y Barris, 1987; Burton, 1989; Allan et al., 2005; Cruz et al., 2014). El trabajo de Burton (1989), por ejemplo, analizó la aplicación del Modelo, principalmente de los factores motivacionales y volitivos, los roles y rutinas, las capacidades cognitivas y los cambios en las habilidades biopsicosociales de la persona mayor en relación con el medio ambiente, estudiando su impacto en el comportamiento ocupacional. Allan et al. (2005), entre tanto, estudiaron el impacto del proceso de jubilación en el desempeño ocupacional, hallando que dicho proceso no provocó el desequilibrio ocupacional y que las personas mayores lograron adaptarse creando una nueva estructura en sus vidas. Por otro lado, Cruz et al. (2014) identificaron como factores protectores de la ocupación en las personas mayores: el trabajo remunerado; la percepción de productividad, competencia y salud; la espiritualidad, y el apoyo del contexto físico y social, los cuales promueven la vida productiva, la calidad de vida y permiten preservar la adaptación ocupacional. Otra investigación en esta línea aplicó el Modelo para indagar los intereses de personas mayores en su comunidad (Yarce et al., 2014).

En este sentido, es pertinente señalar también las orientaciones de organismos internacionales. Por ejemplo, el Informe mundial sobre el envejecimiento y la salud de la Organización Mundial de la Salud (OMS, 2015) promueve la adopción de hábitos y comportamientos saludables para una mejor calidad de vida, a través de políticas de 
salud pública, educación para la salud y disposición de medidas y recursos que puedan adaptarse a todos los países. Estos programas se centran en contribuir para que la creciente población de personas mayores lleve un estilo de vida saludable y participe en actividades significativas. A este propósito pueden contribuir las y los profesionales en Terapia Ocupacional, enfatizando en la ocupación como elemento importante en las estrategias de promoción de la salud y diseñando intervenciones que permitan la participación en ocupaciones significativas (Asociación Americana de Terapia Ocupacional -AOTA, 2008).

Reconociendo los aportes y avances en materia de investigación, teoría, metodología y práctica que han fundado y reconceptualizado las bases del campo de la Gerontología en las últimas décadas (Fernández, 2000; Lalive d'Epinay et al., 2005; Villar, 2005; Baltes et al., 2006; Berger, 2009; Dulcey, 2010; Krzemien, 2013; Ferraro y George, 2016), conviene considerar la influencia de esta renovación paradigmática en la teorización de la Terapia Ocupacional en torno al envejecimiento. En particular, el tránsito del modelo biomédico y de salud-enfermedad al de promoción de la salud y envejecimiento activo. Dentro de este enfoque, la persona mayor es concebida como integrante activa y partícipe de su entorno social, cultural y comunitario (Duque, 2010).

Lo expuesto evidencia que, si bien algunos estudios y prácticas responden a los paradigmas actuales sobre la vejez, como lo proponen Carvalho et al., "el envejecimiento necesita ser pensado y abordado por los terapeutas ocupacionales desde diferentes perspectivas, contemplando también los aspectos so- ciales que involucran a esta población" (2018, p.31). Por lo tanto, siguiendo a estas autoras, se reconoce la necesidad de ahondar en cuestiones colectivas y sociales en la vejez. Por otra parte, a pesar de los avances epistemológicos y teóricos, en la aplicación conceptual y práctica del Modelo de Ocupación Humana en la vejez prevalecen puntos de vista tradicionales, que no incorporan el peso que se le ha dado a los factores contextuales sociopolíticos en sus últimas revisiones (Kielhofner, 2011; Taylor y Kielhofner, 2017).

En suma, esta reflexión parte del reconocimiento de la emergencia de la perspectiva crítica del paradigma social en Terapia Ocupacional, a fin de repensar el enfoque conceptual, la modalidad de abordaje y la aplicación del Modelo de Ocupación Humana en el colectivo de las personas mayores.

\section{El paradigma social en Terapia Ocupacional: su influencia en el abordaje del envejecimiento desde el Modelo de Ocupación Humana}

En Terapia Ocupacional se viene advirtiendo la extensión del enfoque social, ampliando la mirada hacia una perspectiva sociocultural y política que atraviesa y da sentido a las intervenciones en los diferentes campos (Simó et al., 2016). Conviene, entonces, reflexionar sobre el abordaje del envejecimiento desde un paradigma que integre aspectos micro y macrosociales como la participación socio-comunitaria, los derechos humanos, los movimientos sociales, las políticas públicas, los microemprendimientos no gubernamen- 
tales, las diversas configuraciones familiares, es decir, las prácticas sociales, reconociendo a la persona adulta mayor como participante activa de la sociedad y como ciudadana.

Actualmente, la Terapia Ocupacional transita por lo que autores como Morrison (2018) han denominado el paradigma social o cuarto paradigma, que dio lugar a la perspectiva social y comunitaria de la profesión. Este enfoque aporta ideas para la comprensión del envejecimiento como fenómeno social y colectivo, es decir, como un producto configurado por múltiples factores sociales, culturales y económicos que determinan la participación de la persona mayor en su entorno. A su vez, permite considerarla como sujeto de derechos, actuante, productora y transformadora de su medio (Morrison et al., 2016; Carvalho et al., 2018). De esta manera, el paradigma social de la ocupación proporciona el fundamento teórico para desarrollar prácticas e intervenciones efectivas que fomenten su participación social (Turcotte et al., 2018; Smallfield y Molitor, 2018).

No obstante, varios autores argumentan que la participación ocupacional a menudo se ve restringida por opiniones convencionales sobre el envejecimiento, situadas en un contexto político neoliberal (Simó et al., 2016; Trentham y Neysmith, 2018). Dichas concepciones moldean la forma en que las personas mayores ejercen su ciudadanía, limitando sus posibilidades para ejercer roles ocupacionales. De esta manera, es relevante generar un mayor conocimiento que conduzca al cambio social en este terreno.

Por otra parte, la dimensión política de la Terapia Ocupacional ha tenido especial protagonismo a partir de su aceptación por parte de la Federación Mundial de Terapeutas Ocupacionales en su reunión de Consejo Mundial celebrada en el 2004 en Sudáfrica. Luego de una intensa polémica, la Federación decidió tomar una postura oficial sobre la incorporación de conceptos como apartheid ocupacional, privación ocupacional y justicia ocupacional (Kronenberg et al., 2005), lo cual dio pie a que las y los terapeutas ocupacionales incluyeran en sus intervenciones cuestiones relacionadas con la ciudadanía y los derechos humanos. Brasil fue uno de los países precursores de este paradigma, con trabajos como la visión crítica de la Terapia Ocupacional de Berenice Francisco (1988), y permanece a la vanguardia de estos enfoques.

Habiendo superado una mirada ahistórica y reduccionista de la ocupación, la incorporación de los aspectos sociales, culturales y políticos permite, como postula Guajardo (2012), entender las ocupaciones como prácticas sociales en las cuales se producen relaciones que dan lugar a la conformación de sujetos como un suceso totalizador; la ocupación no es un hecho aislado, en el decir del autor, somos la ocupación. Por su parte, Galheigo (2012) destaca el concepto de cotidiano como un acontecer histórico que refleja, entre otros, los valores, sentimientos e ideas del sujeto $y$, a partir del cual, no solo arriba al conocimiento de la sociedad en la que está inmerso, sino a la posibilidad de transformación social; de esta manera, se supera la visión positivista de las actividades de la vida diaria. La Terapia Ocupacional debería posibilitar, entonces, prácticas que incluyan tanto el ejercicio de la ciudadanía como de los derechos.

En relación con el Modelo de Ocupación Humana, Kielhofner (2011) se- 
ñala que este es producto de más de tres décadas de conceptualización, investigaciones y aplicaciones prácticas. Si bien en un comienzo estaba diseñado para orientar a las personas con discapacidades que tenían limitaciones en su participación ocupacional, el propio autor propuso una reformulación teórica que se expone en la cuarta edición de su libro Modelo de Ocupación Humana (Kielhofner, 2011) ${ }^{4}$ y en su capítulo del libro Terapia Ocupacional sin fronteras (Abelenda et al., 2006). Para entonces, Kielhofner reconoció que los nuevos estudios remarcaban la importancia de contribuir al cambio social tanto individual como de grupos, colectivos y organizaciones, para las y los terapeutas ocupacionales (Abelenda et al., 2006). Este replanteamiento inauguró una perspectiva más sociopolítica y contextual, vinculada a las problemáticas sociales.

Kielhofner (2011), y recientemente De las Heras (2015), redefinieron el concepto de medio ambiente, para referirse a entorno, sumando a las dimensiones existentes, las influencias de las condiciones políticas y económicas para el análisis de la participación ocupacional. En este sentido, Abelenda et al. (2006) destacan que, para entender el fenómeno ocupacional en su complejidad social, económica y política, se requiere de una mirada multidisciplinaria de los sistemas sociales.

Por otra parte, la perspectiva brasilera prefiere referirse a actividades humanas, más que a ocupación, como objeto de estudio de la Terapia Ocupacional (Da Rocha Medeiros, 2008; Galheigo, 2012; Carvalho, 2018). Quienes conciben la ocupación humana como actividades humanas, desde diversos contextos y regiones, reconocen el carácter complejo, multifacético y dinámico de la naturaleza ocupacional a lo largo del curso vital (Da Rocha Medeiros, 2008; Kielhofner, 2011; Galheigo, 2012; Guajardo, 2012; De las Heras, 2015; Carvalho, 2018).

Adhiriendo a esta perspectiva, en este texto se considera que el Modelo de Ocupación Humana, junto con otros enfoques, pueden dar respuesta a una visión gerontológica que haga mayor énfasis en las variables contextuales, lo cual resulta necesario para abordar las cuestiones actuales del envejecimiento

\section{Modelo de Ocupación Humana: derivaciones teóricas en la comprensión del envejecimiento}

Con el interés de extender y profundizar en la concepción de la vejez como proceso del curso vital humano desde el Modelo de Ocupación Humana (Kielhofner, 2011), se hará referencia a los principales postulados teóricos de este modelo en cuanto al cambio y desarrollo ocupacional en la vida adulta avanzada: de qué modo la ocupación es motivada, cómo se organiza en patrones de vida y cómo se desarrolla el desempeño ocupacional en el contexto del entorno.

\section{Ocupación y adaptación ocupacional}

La ocupación humana se entiende como la participación en actividades mediante las cuales se van desarrollan-

${ }^{4}$ Publicado originalmente en 2008 y traducido al español en el 2011. 
do las experiencias ocupacionales de las personas. La variedad de experiencias dará como resultado la adaptación ocupacional y sus componentes, la identidad y la competencia. Así, la historia de participación social en actividades significativas de la persona mayor construye su identidad, así como su competencia y adaptación ocupacional (Krzemien y Lombardo, 2003; Kielhofner, 2011).

El concepto de adaptación ocupacional es concebido por Kielhofner (2011) como una resultante de la historia de participación ocupacional y de la interacción entre la persona y su entorno, en la medida en que se construye con el tiempo una identidad ocupacional positiva y se logra competencia ocupacional en el contexto del medio ambiente. Esta adaptación, lejos de ser lineal o estática, es dinámica, mostrando avances y retrocesos de acuerdo con la manera en la que las experiencias modelan los comportamientos. Según Palma et al. (2019), en personas mayores el proceso de adaptación ocupacional exitoso está asociado con la construcción de una nueva identidad ocupacional, con un sentido de eficacia y competencia, y con el compromiso de participar en una ocupación significativa. Por su parte, Johansson y Björklund (2016) resaltan que las personas mayores que participaron en un programa de promoción de la salud en viviendas comunitarias, incluyendo actividades ocupacionales, mostraron mejoras estadísticamente significativas en variables generales de salud como la vitalidad, la salud mental y el bienestar psicológico. Las investigadoras concluyen que la participación en actividades significativas y desafiantes en diferentes ambientes estimula el proceso de adaptación ocupacional, mejorando la vida de las personas mayores.

Una de las ideas centrales en la concepción del Modelo de Ocupación Humana es el énfasis en la acción, en el hacer ocupacional; no se trata solo de la persona que realiza una actividad ocupacional, sino del sentido en que la acción define a la persona y da significado a su trayectoria de participación, en interacción con su entorno. En este sentido, De las Heras (2015) enfatiza el hacer como concepto clave del Modelo, definiéndolo como "explorar y practicar la propia capacidad de experimentar, responder y resolver situaciones diarias" (p.34). A su vez, esta autora incluye en las dimensiones de participación en ese hacer, el "experimentar, a través del sentir, el contexto ocupacional en relación consigo mismo" (p.43).

Desde esta perspectiva, Kielhofner (2011) entiende la ocupación humana como "la realización ${ }^{5}$ del trabajo, el juego o las actividades de la vida diaria dentro de un contexto temporal, físico y sociocultural, que caracteriza gran parte de la vida humana" (p.5) y "la acción que ocupa un lugar particular, social y físico" (p.97). Es justamente en esta participación, en sus diversas modalidades y en los diferentes contextos en los que ha interactuado la persona mayor, donde se han ido moldeando la volición, la habituación y la capacidad de desempeño; por ende, de ese quehacer resultan las posibilidades de adaptación ocupacional. A continuación se hará referencia a estos tres componentes en la vejez.

${ }^{5}$ Doing, en el idioma original del autor. 


\section{La volición}

A medida que transcurre la vida, los pensamientos y sentimientos volitivos se van moldeando en función de los valores propios y los intereses ocupacionales que se han conformado en relación con el contexto de interacción social y las posibilidades de desarrollo o restricciones en el ambiente. En otras palabras, el entorno puede proveer oportunidades y recursos de elección, o bien, puede limitar o generar restricciones en la selección o planificación de los cursos de acción. Por ejemplo, la pandemia global causada por el COVID-19 ha condicionado los entornos y los estilos de vida de las personas, en particular de las mayores, restringiendo las posibilidades ocupacionales y la socialización a través del contacto físico; al mismo tiempo, se han ampliado en el medio cultural las posibilidades de interacción social mediante la virtualidad.

Causalidad personal. Cuando el entorno de una persona mayor limita su desenvolvimiento funcional y su desarrollo en algún área de la vida ${ }^{6}$, se pueden ver afectadas las capacidades propias y el sentido de eficacia personal, es decir, la causalidad personal. La evidencia demuestra que, en estas personas, la autoeficacia está relacionada con el nivel de participación ocupacional. Por ejemplo, Peterson et al. (1999) encontraron que las y los adultos mayores que han sufrido caídas exhiben una disminución de la autoeficiencia y, por ende, una reducción en la participación en ocupaciones de recreación y sociales. En este caso, la experiencia negativa puede alterar el propio sentido de competencia y efectividad, independientemente del riesgo real de volver a sufrir una caída y de los antecedentes de incidentes similares; así puede afectarse la apreciación cognitiva que hace la persona de sus propias capacidades y recursos, restringiendo su participación, aumentando el riesgo de futuras caídas y afectando su capacidad funcional de adaptación.

Por el contrario, la relación entre la autoeficacia y el nivel de participación ocupacional en personas mayores puede analizarse desde el envejecimiento saludable o competente. En este sentido, alguna literatura gerontológica alude a la perspectiva del envejecimiento con éxito: saludable, activo, productivo, óptimo y positivo (Fernández, 2007; Fernández et al., 2010). Estas nociones surgen de la variabilidad interindividual y de las diversas formas de envejecer en nuestra sociedad actual. El concepto de envejecimiento exitoso o competente es congruente con una valoración subjetiva de la experiencia vital y del bienestar psicológico, definiéndose como conformidad o satisfacción con la vida y una combinación de vitalidad, resistencia, flexibilidad adaptativa, integridad, control y autonomía, y un ambiente social facilitador del cambio y el desarrollo. En concordancia con estas ideas, Kielhofner (2011) refiere que muchas personas mayores pueden ser sumamente creativas para mantener un sentido de eficacia; así, ante circunstancias adversas, responden activamente para evitar un resultado no deseado, redireccionar su vida y continuar participando en ocupaciones significativas (Jonsson et al., 1997).

\footnotetext{
${ }^{6}$ Por ejemplo, desde lo social, mediante el prejuicio o la discriminación debido a la edad, o desde el punto de vista físico, en el hábitat.
} 
Ahora bien, estas ideas no pueden dejar de contemplar la compleja interacción entre los aspectos individuales, contextuales y ambientales en el proceso de desarrollo del curso de vida. Así, el carácter exitoso en el envejecimiento no se restringe a una cuestión del individuo, sino más bien al entramado de las múltiples variables sociales. De esta manera, conviene pensar la volición como un proceso en el que intervienen aspectos personales cognitivos (cuando uno anticipa, selecciona, planifica e interpreta) y aspectos contextuales de la experiencia ocupacional. En consecuencia, tanto los conceptos de causalidad como el sentido de eficacia en una persona, lejos de ser individuales, son influenciados por las variables contextuales.

En este sentido, Rudman (2015) encontró en el discurso de personas en proceso de preparación para la jubilación, la incorporación de contenidos positivos junto con una racionalidad neoliberal, en especial referida a los cuidados y la sensación de control corporal. La autora explica los resultados en función de un contexto neoliberal en el que la salud, la sociedad, las finanzas y otras responsabilidades se desplazan a los individuos. A su vez, Rubinstein y de Medeiros (2015), en su crítica a la perspectiva del envejecimiento exitoso de Rowe y Kahn (1987), señalan que esta sobrevalora la acción individual como el principio de motivación más importante para alcanzar el éxito, exhibiendo un lenguaje que sugiere que aquellas personas que envejecen sin éxito o que, en otras palabras, fracasan, lo hacen por responsabilidad propia. Este enfoque, por lo tanto, carece de un análisis complejo que considere las variables económicas, sociales, culturales y políticas que fundamentarían el logro del envejecimiento exitoso.
La valoración y los valores en la vejez. Si bien la transformación de los valores a lo largo de la vida es reconocida en el Modelo de Ocupación Humana, esta no necesariamente se relaciona con cada etapa. Más bien, la pluralidad de estilos de vida y de elecciones ocupacionales de las personas adultas y adultas mayores, los cambios socioculturales, la variabilidad interindividual en las elecciones e intereses, desdibujan y desligan la estandarización de cursos de vida en relación con los valores según el criterio etario. Es decir, la edad o la etapa vital de una persona no determinan la identificación de valores. Esta idea se refleja en la diversidad de roles que en la actualidad asumen las y los adultos mayores, acorde con las transformaciones socio-históricas que han ido experimentando, lo cual muestran que las elecciones y la participación ocupacional dependen cada vez menos de cursos de acción institucionalizados. Se reconoce la singularidad de las historias de vida, en las cuales, la naturaleza y la dirección de cambio de valores varía de acuerdo con las condiciones y el impacto del entorno. La percepción de los recursos, el apoyo, las demandas o restricciones ambientales, así como la trayectoria ocupacional y los aspectos volitivos personales influyen en los valores asumidos. De esta forma, los valores pueden mantenerse o transformarse $y$ continúan siendo fundamentales en las elecciones ocupacionales de las personas mayores (Kielhofner, 2011).

Algunos estudios muestran que los valores relacionados con los vínculos conyugales, familiares y de pares, la comunidad y la recreación parecen ser los más relevantes en la vejez (Antonovsky y Sagy, 1990; Krzemien, 2006). Aunque tradicionalmente se concibe que, 
asumida la vejez, los valores se modificaban de lo activo a lo pasivo; de lo productivo, el logro, la ambición y la eficiencia hacia valores como la condescendencia, la comodidad, la sabiduría, la consejería, entre otros (Kielhofner, 2011, p.136), la transición de los valores en la vejez es compleja, dado que depende de los cambios culturales, políticos, socio-históricos y demográficos, así como de los avances en el campo epistemológico, teórico y metodológico de la Gerontología. Además, considerando la singularidad de las historias de vida, la idiosincrasia y las circunstancias contextuales, los valores asumidos por las personas mayores son variados y resignificados.

Los intereses. Para las personas mayores, el albedrío y el aumento del tiempo exento de responsabilidades laborales constituyen una oportunidad para dedicarse a variados intereses de forma más plena y para ampliar el horizonte de aficiones. Así mismo, resultan importantes las posibilidades que brinden los entornos físicos y sociales, pues pueden facilitar o restringir la participación ocupacional. Según Miranda, el comportamiento para involucrarse en actividades cotidianas significativas

Responde, por una parte, a las necesidades individuales y al sentido de gratificación que cada persona asigna a las diferentes ocupaciones. Por otra (...), a las alternativas de exploración y a la forma particular de interacción, condicionadas al medio sociocultural, económico y político en el cual se halla inmerso el adulto mayor. (2005, p.210)

De esta manera, es posible replantear el concepto de tiempo libre u ocio en la vejez, reconociendo que en el contexto actual, a menudo las personas mayores se involucran en actividades significativas y productivas que tienen un impacto socioeconómico directo para las comunidades, como en situaciones de escasez de recursos, coyunturales o migratorias en las que asumen roles protagónicos como proveedores o cuidadores (Horstead y Bluestone, 2018).

Otro concepto que cobra relevancia para abordar los intereses en la vejez es el de ocupación motivante, el cual hace referencia a que la elección de una ocupación suponga un fuerte sentimiento o pasión por realizarla, se relacione con los intereses y la causalidad personal, desafíe la propia competencia y se reconozca como valorada, de modo que signifique una contribución a la familia o a la sociedad y conduzca a una participación regular a lo largo del tiempo (Kielhofner, 2011). Es el caso de las personas mayores, para quienes emprender y sostener una actividad, por lo general, ya no conlleva un sentido de obligatoriedad ni una elección en función de convenciones sociales o de reconocimiento público, sino el interés significativo y motivador propio. A su vez, la variable tiempo y su percepción adquieren una dimensión y un valor particular en esta etapa, como motivador de las elecciones ocupacionales, organizando una jerarquía de prioridades en las metas vitales propuestas.

\section{La habituación en la vejez}

La habituación, entendida como patrones constantes y aprendidos de comportamiento, comprende los hábitos y los roles ajustados a las características del ambiente, dando lugar a rutinas de actividades (Kielhofner, 2011). Los cam- 
bios culturales, el impacto de eventos de vida críticos, las demandas sociales y la diversidad de información en nuestro tiempo y culturas, desafían la regularidad de los hábitos y costumbres aprendidos en el transcurso de la vida; a su vez, ello posibilita una mayor flexibilidad en la modificación de patrones. Las personas mayores se enfrentan a cambios en su capacidad de adaptación a la habituación.

Por otra parte, las etapas vitales no definen los hábitos y roles de las personas, es decir, no hay hábitos y roles específicos o propios de la vejez. Los roles que tradicionalmente se han atribuido a las personas mayores, por ejemplo, el de jubilada(o) o abuela(o), en la actualidad se redefinen; hoy las observamos como estudiantes en universidades, trabajando en emprendimientos propios, ocupando roles de gerencia, de experticia y de gestión, de ejercicio de ciudadanía, iniciando una nueva pareja después de la viudez, asumiendo una paternidad longeva, entre otros. En las políticas y programas relacionados con el envejecimiento a nivel local, nacional e internacional, se evidencia también la participación ocupacional en nuevos roles; por ejemplo, en las últimas décadas se han potenciado los programas universitarios para mayores. Estos programas apuntan a mejorar integralmente la calidad de vida de las personas mayores, ya que no responden a concepciones reduccionistas que se limitan a la generación de espacios socio-recreativos (Yuni y Urbano, 2016). La tradicional propuesta de talleres o actividades para este grupo remitía a una perspectiva bioevolutiva o a un enfoque biomédico de evitar las pérdidas asociadas a la edad, prevenir enfermedades neurodegenerativas o, simplemente, estimular la memoria; o bien, se enfocaba en planes de recreación y actividades asociadas a roles de abuelidad. Por el contrario, la propuesta de los programas universitarios para mayores se diferencia en que estos lugares permiten su integración en diferentes ámbitos de la comunidad, estimulan el ejercicio de sus derechos ciudadanos y presentan una oferta educativa diversa, que responde a una amplia gama de intereses.

Ahora bien, como los hábitos constituyen aprendizajes de esquemas de comportamiento y organizan las rutinas en la vida cotidiana, en algunos casos las personas tienden a aferrarse a ellos, lo que limita sus posibilidades de aprendizajes nuevos. Quienes logran superar esos condicionamientos de habituación tienen mayor capacidad para adaptarse a situaciones nuevas y desafiantes. Sin embargo, el desarrollo relativamente estable de hábitos y roles a lo largo del curso vital, en diferentes áreas ocupacionales de la persona mayor, tiende a afianzar la conservación de la habituación (Kielhofner, 2011). Esta adaptación resulta útil en aquellas personas de edad avanzada que presentan diversas patologías psicofísicas o cuyas capacidades funcionales están afectadas, quienes dependen en mayor medida de la contención familiar, la comunidad y las instituciones para mantener su desempeño ocupacional y su calidad de vida. En cambio, en personas sin patologías y cuyo entorno proporcione condiciones políticas, socioculturales y económicas favorables, las oportunidades de desempeñar roles diversos y de modificar la habituación pueden generar una mayor flexibilidad en la organización de las rutinas cotidianas, empoderándolos en la elección de roles de participación ocupacional y, por ende, optimizando su capacidad adaptativa. En síntesis, las personas mayores suelen desarrollar há- 
bitos a lo largo de los años en un entorno estable y reconocido, por ello, los cambios en el contexto físico y sociocultural son un desafío, en tanto las capacidades físicas y psicológicas también se modifican. De este modo, la flexibilidad en la asunción de hábitos y roles es más relevante a medida que avanza la edad, para sostener e incrementar el desempeño funcional y la apreciación cognitiva de bienestar y calidad de vida.

\section{Capacidad de desempeño}

Esta se define como la capacidad para el hacer, provista por la condición de los componentes objetivos físicos y mentales y la experiencia subjetiva correspondiente (Kielhofner, 2011). Este autor afirma que "en el curso de nuestra vida ocupacional diaria, nuestro cuerpo es un punto de vista invisible desde el cual experimentamos y actuamos en el mundo" (2011, p.71). En la vejez, este concepto cobra especial relevancia para comprender la capacidad adaptativa en términos del desempeño ocupacional, la realización de actividades y el rendimiento psicocognitivo, donde se involucran cambios en los aspectos neurobiológicos, psicomotor, socioemocional y neurocognitivo.

El Modelo de Ocupación Humana reconoce dos dimensiones de esta capacidad de desempeño: una objetiva, relativa a los diferentes sistemas biológicos, corporales y neuroanatómicos, entre otros, y otra subjetiva, la experiencia de ser y conocer el mundo en el ejercicio de las capacidades. En este aspecto, la mente y el cuerpo son reconocidos como parte de una entidad única. Kielhofner (2011) se refiere a la noción de cuerpo vivido como la experiencia subjetiva del ejercicio de las capacida- des de la persona. En la vejez, cuando algunas habilidades tienden a declinar y se van afectando ciertas capacidades, la capacidad objetiva de rendimiento cognitivo puede no ser tan eficiente como en el pasado; no obstante, en condiciones favorables, la experiencia subjetiva del hacer y del desempeño ocupacional puede conservarse y aún incrementarse. Con los aportes de las neurociencias acerca de los cambios del cerebro envejecido (Harris y Allegri, 2009; Allegri et al., 2010; Soto y Arcos, 2010; Redolar, 2014) es posible explicar cómo la neuroplasticidad de las redes neuronales, en relación con las capacidades de reserva cognitiva, permiten que la persona mantenga su capacidad funcional por un mecanismo cerebral compensatorio (Stern, 2011; Arenaza y Bartrez, 2014; Jones et al., 2014), ejerciendo su desempeño ocupacional de manera relativamente eficiente. A su vez, respecto al componente subjetivo, en el caso de un proceso patológico o discapacitante, las personas tienen que llegar a comprender, transformar o aprender nuevas formas de experiencias de desempeño ocupacional.

\section{Los entornos y su condicionamiento en la vejez}

Como se ha expuesto, los tres componentes que, según el Modelo de Ocupación Humana, conforman la naturaleza ocupacional de las personas (volición, habituación y capacidad de desempeño) están influenciados por las particularidades del entorno donde se desarrollan. En palabras de Kielhofner, "las características particulares físicas y sociales, culturales, económicas y políticas de los contextos personales impactan en la motivación, la organización y el desempeño de la ocupación" (2011, 
p.86). Así, tales condiciones pueden favorecer o restringir las oportunidades de elección y desarrollo ocupacional de las personas mayores.

Para comprender la ocupación en el envejecimiento conviene entender la realidad social, comunitaria, cultural, material y simbólica en la que las y los adultos mayores viven; ello es fundamental para dar respuestas coherentes con sus necesidades en el contexto social (Cabral et al., 2018; Carvalho et al., 2018). La acción de los organismos internacionales y regionales en materia de envejecimiento y sus recomendaciones, así como las periódicas reuniones internacionales sobre el tema han promovido políticas para propiciar entornos favorables o ciudades amigables con las personas mayores (Organización Iberoamericana de Seguridad Social -OISS, 2016; Leal, 2017; Pozo, 2017), insistiendo a los gobiernos, sobre todo aquellos en países que presentan un mayor envejecimiento poblacional, en la necesidad de comprometerse con programas y políticas públicas que garanticen su participación ocupacional y comunitaria en el entorno particular en el que se desenvuelven. Sin embargo, en algunas sociedades, los conflictos políticos y las injusticias económicas restringen o inhiben la vida ocupacional de las personas de edad avanzada que son víctimas de esas condiciones de deprivación e indefensión social (Kielhofner, 2011). Es así como las condiciones negativas o positivas de los entornos interactúan con los componentes personales e impactan sobre las posibilidades de participación ocupacional significativa y satisfactoria de las personas mayores.

\section{Construcción de la vida ocupacional: identidad, competencia y adaptación ocupacional en adultos mayores}

Se ha mencionado ya que uno de los conceptos clave en el Modelo de Ocupación Humana para comprender la construcción de la vida ocupacional, particularmente en el envejecimiento, es el de adaptación ocupacional. Esta se relaciona con la historia de participación en una variedad de actividades y experiencias en un contexto de interacción psicosocial donde se van conformando los componentes de esa adaptación: identidad ocupacional y competencia (Kielhofner, 2004, 2011).

En las personas mayores se produce una reconstrucción y renovación del sentido de identidad propia, a partir de la historia de participación en un contexto donde se redefinen las dimensiones personales (volición, habituación y capacidad de desempeño) en relación con las experiencias de cambio, tanto limitaciones y pérdidas como progresos y ganancias. De esta manera, se reconstruyen: a) la percepción de la propia capacidad y eficacia para hacer -causalidad personal; b) lo que se vive como satisfactorio y gratificante -intereses; c) lo que se reconoce cultural y subjetivamente como importante y las convicciones de vida -valores; d) los roles y rutinas que se desarrollan -habituación, y e) la regulación dinámica de las capacidades, habilidades y destrezas psicofísicas y neurocognitivas, de manera que la persona puede afrontar los cambios propios del envejecimiento y del ambiente que le rodea. La reconstrucción positiva de la percepción de es- 
tos aspectos de la identidad ocupacional se reflejará en una modificación adecuada del patrón de participación, esfuerzo y logro que supone la competencia ocupacional. Kielhofner (2011) concibe que, a medida que las personas se esfuerzan por organizar sus desempeños en rutinas de comportamientos competentes y relevantes para sí mismas y para el entorno, se van convirtiendo (en el sentido de cambio personal), creciendo y llegando a un mayor sentido de eficacia. Este proceso de conversión, en tanto pasaje por una serie de etapas en una continuidad dinámica e interrelacional, involucran la exploración, la competencia y el logro en el transcurso vital.

\section{El rol de la narrativa en la adaptación ocupacional en el envejecimiento}

Kielhofner (2011) plantea que la construcción de la identidad y la competencia ocupacional durante el curso vital se expresa mediante una narrativa que es moldeada por la cultura: "si bien cada persona construye su propia narrativa, el contexto sociocultural tiene una importante influencia" (p.1123) Al respecto, el autor formula algunos interrogantes:

\begin{abstract}
¿De qué modo nos las arreglamos para elegir entre los muchos mensajes, expectativas y oportunidades de nuestros medios ambientes?, ¿cómo seleccionamos qué hacer luego y cómo tenemos el sentido de dónde encaja? y ¿cómo llegamos a manejar nuestras vidas y nos arreglamos para vivirlas? (Kielhofner, 2004, p.142).
\end{abstract}

Las respuestas a estas preguntas podrían ser argumentadas, en parte, reconociendo que las personas orientan sus elecciones en función de los signi- ficados culturales que dan sentido a las prácticas sociales y a las actividades cotidianas, y se estructuran en narrativas que integran nuestro ser pasado, presente y futuro (Gergen y Gergen, 1988; Jonsson et al., 2001).

Dos aspectos relevantes para la comprensión de estas narrativas son el argumento y la metáfora, los cuales le otorgan sentido a las situaciones y episodios. El argumento se refiere a la estructura de la narrativa, en función del significado general que resume dónde ha estado y hacia dónde está yendo la vida, atribuyéndole valoraciones a los eventos en la medida que fluyen a través del tiempo en un continuo donde, en un momento determinado, se aprecian de manera positiva o negativa.

Respecto a la metáfora, Schön (1979), en su análisis del lenguaje y el discurso, introduce el concepto de metáfora generativa para referirse tanto a un tipo de producto (una perspectiva o marco, una forma de ver las cosas) como de proceso mediante el cual surgen nuevas perspectivas sobre el mundo. La metáfora es utilizada en la narrativa como generadora de nuevos significados para las circunstancias o eventos de la vida que son especialmente difíciles de comprender o de enfrentar, a fin de integrarlos al transcurso de la vida ocupacional y que tengan efectos en la adaptación ocupacional. Por ejemplo, metáforas como luchar la batalla o ganar la guerra, en el caso de las enfermedades.

Para el Modelo de Ocupación Humana, "las personas comprenden su vida ocupacional en términos de una narrativa y buscan influir en el modo en que se despliega su narrativa ocupacional" (Kielhofner, 2011, p.511). Es 
decir, los seres humanos otorgan sentido y significado a sus experiencias ocupacionales mediante narrativas. El autor alude a la idea de narrativa ocupacional para comprender la forma en la que las personas significan sus experiencias de vida y analizan las circunstancias nuevas que se les presentan, evaluando cómo acontecimientos similares se han desarrollado antes y hacia dónde pueden conducirlas ahora. A través de la construcción de narrativas, las personas encuentran sentido a los episodios de la vida y anticipan el futuro.

La narrativa ocupacional es tanto la historia contada como experimentada y anticipada, que integra la volición, la habituación y la capacidad de desempeño en los entornos en los que se despliega a través del tiempo. La historia de cada persona refleja sus metas, proyectos, desafíos, logros; es decir, los componentes de la identidad y la competencia se entretejen en esas historias. Estas narrativas pueden graficarse mediante pendientes que ilustran las diferentes direcciones que pueden tomar, en tres sentidos: progresivo (mejora), estable (se mantiene igual) y regresivo (empeora) (Jonsson et al.,1997). Al respecto, un estudio longitudinal con personas mayores en periodo pre y posjubilación mostró que la orientación de las pendientes de sus narrativas tenía como referente la experiencia laboral previa, y que su vida ocupacional se expresaba en narrativas moldeadas por los episodios vividos y por aquellas vivencias que no se anticiparon al momento de jubilarse (Jonsson et al.,1997; Jonsson et al., 2000).

En referencia a la construcción discursiva en el envejecimiento, en las personas mayores los cursos de vida y los eventos vividos adquieren diferen- tes formas según cada contexto histórico-social. ¿Cómo las trayectorias de vida son narradas por diferentes personas? ¿De qué forma éstas son imaginadas, significadas, producidas discursivamente e internalizadas? ¿Cómo llegan a reproducirse y hacerse reales en la vida cotidiana? Son algunos de los tópicos de interés que, hasta el momento, han sido poco atendidos por la Gerontología (Krzemien, 2013). Por ello, es relevante profundizar la investigación de estas cuestiones desde la perspectiva del Modelo de Ocupación Humana.

El curso de la vida es atravesado por valores, creencias y representaciones compartidas socialmente, presentes en un determinado contexto histórico-social, y es influenciado por aquello que socioculturalmente es esperable para el grupo etario de las personas mayores. En la medida que la persona mayor interioriza esas representaciones sociales, forman parte de su subjetividad, dando forma a su identidad y competencia, y vehiculizándose en su narrativa ocupacional. Cada narrativa contiene significaciones de la cultura y del contexto particular, las cuales suelen ser diversas, contradictorias, desarticuladas, según las experiencias de vida y su relación con cada grupo de pertenencia. En este sentido, los argumentos de la narrativa pueden tener carácter regresivo, progresivo o estable (Kielhofner, 2011), de acuerdo con la evaluación y la apreciación cognitiva que la persona hace de los eventos de su vida en términos de su significado y del impacto en su historia.

La vida ocupacional en la vejez adquiere un carácter multifacético, dada la participación en diferentes roles y áreas ocupacionales a lo largo de cada historia, lo cual aumenta la variabilidad 
interindividual de este grupo etario; esta heterogeneidad en la participación ocupacional se evidencia en las diversas narrativas, tanto en cómo se estructuran los argumentos como en la generación de metáforas. Tales narrativas revisten una naturaleza especialmente subjetiva, de acuerdo con la particularidad e idiosincrasia de las significaciones de la propia historia de vida, pero a la vez contienen elementos de los temas sociales predominantes, articulándose con las representaciones socioculturales acerca del envejecimiento y la vejez vigentes en el contexto. Tradicionalmente, estas representaciones estaban vinculadas a ideas y valoraciones estereotipadas y restringidas sobre las capacidades y el sentido de autoeficacia, lo cual limitaba los roles y la participación ocupacional de las personas mayores. Actualmente, en las narrativas de las personas mayores se observa la selección de una mayor diversidad de áreas de participación ocupacional, incluso asumiendo roles que en el pasado eran asignados a otras etapas de la vida. Entonces, en una misma narrativa es posible encontrar diferentes pendientes que marcan una trayectoria más dinámica, por momentos regresiva, por otros, progresiva o estable. Esta multiplicidad de significaciones y de pendientes de trayectorias puede, en algunos casos, otorgar una mayor flexibilidad en la adaptación ocupacional.

\section{Las narrativas ocupacionales en la vejez desde la perspectiva crítica contemporánea}

En el marco del pensamiento crítico contemporáneo, no solo se valoriza el papel del lenguaje en la construcción del conocimiento, sino el de la acción, el de las prácticas sociales como formas de expresión de conocimiento. Es decir, se trata de pensar al lenguaje, no como un compendio de significados o en su valor lingüístico, sino como un instrumento mediador de las relaciones entre las personas. En este sentido, recordemos que Vigotsky (1979), en su prolífica y original obra, plantea la actividad social humana como significativa al estar mediada semiótica y simbólicamente por el lenguaje como instrumento propio de la cultura. Esto revela la constitución de un contexto en el que intervienen intenciones, convenciones, procesos sociales de negociación y multiplicidad de sentidos dados. Las realidades sociales son significados producidos en la interacción en un contexto determinado (Bruner, 1990). En efecto, el contexto sociocultural condiciona cierta clase de experiencias a partir de las cuales se genera el discurso narrativo, siendo el lenguaje social un vehículo consensuado que da validez a cada una de las producciones simbólicas. Shotter (2001) entiende el discurso en su capacidad constructiva de relaciones sociales; lo que se habla cobra significado en el contexto de esas relaciones construidas de manera dinámica y sostenida.

Una de las premisas centrales del enfoque crítico es la interpretación de la multiplicidad de significados de la experiencia humana en un contexto de interacción social y cotidianeidad. De hecho, es materia de investigación comprender cómo las personas mayores crean concepciones y mantienen determinadas formas de relacionarse entre sí, y cómo a partir de esa multiplicidad de formas de comunicarse, interpretan sus circunstancias y acontecimientos. De esta manera puede recuperarse la dimensión de la vida cotidiana de las personas mayores y es posible enfatizar en la mirada idiosincrásica del curso vital, resaltando sus propias voces y los significados 
subjetivos atribuidos a su experiencia, de manera que sean más comprensibles las experiencias vividas (Gubrium, 1993; Calasanti y King, 2015).

Esta perspectiva resulta apropiada para el estudio de la narrativa acerca de la participación ocupacional en la vejez y para abordar la heterogeneidad de las trayectorias de vida y sus implicaciones en la adaptación ocupacional. Considerando a Galheigo (2012), la dimensión de la vida cotidiana de las personas mayores, en la medida que se expresa en una narrativa ocupacional, puede ser analizada desde la Terapia Ocupacional, reconociendo la voz subjetiva en un acontecer temporal e histórico del transcurrir del envejecimiento.

En consecuencia, resulta de interés profundizar en el conocimiento de las narrativas ocupacionales colectivas, que permitan analizar las transformaciones sociales, en pos del bienestar de comunidades de personas mayores. En este sentido, el concepto de adaptación ocupacional puede ser repensado, lejos de referirnos a una adecuación al sistema social vigente, como la reconstrucción de las diversas identidades ocupacionales y relaciones sociales de las personas mayores, dando lugar a un empoderamiento como colectivo, con un sentido de mayor solidaridad y justicia ocupacional.

\section{Conclusión}

Esta reflexión crítica se orientó a contribuir a la actualización de las concepciones teóricas del Modelo de Ocupación Humana en la comprensión de la ocupación en la vejez, considerando la perspectiva contextual y del paradigma del curso vital. A partir de estas ideas, la aplicación del Modelo conlleva a enfocar la práctica profesional gerontológica en función de las condiciones sociopolíticas y contextuales que afectan la adaptación ocupacional, la participación social y las narrativas volicionales del colectivo de personas mayores. Esta perspectiva permite que la intervención en Terapia Ocupacional transite desde la mirada asistencial hacia la consideración de la persona mayor como agente social, activa y ciudadana en su comunidad. Sería pertinente sumar nuevos esfuerzos de teorización e investigación en el campo de la Terapia Ocupacional en Gerontología, a fin de renovar el diseño de programas de intervención terapéutica y de promoción de la salud y la calidad de vida de las personas mayores.

\section{Referencias}

Abelenda, J., Kielhofner, G. y Suarez, Y. (2006). El modelo de ocupación humana como herramienta conceptual para comprender y abordar la segregación ocupacional. En F.Kronenberg, S. Simó y N. Pollard (Eds.), Terapia ocupacional sin fronteras: Aprendiendo del espíritu de los sobrevivientes (pp. 83-196). Editorial Médica Panamericana.

Allan, A. N., Wachholtz, M. D. y Valdés R. A. (2005). Cambios en la ocupación de los adultos mayores recientemente jubilados. Revista Chilena de Terapia Ocupacional, 5, 19-29. https:/doi.org/10.5354/0717$\underline{5346.2005 .103}$

Allegri, R. F., Taragano, F. E., Krupitzki, H., Serrano, C. M., Dillon, C., Sarasola, D., Feldman, M., Tufro, G., Martelli, M. y Sánchez, V. (2010). Role of cognitive reserve in pro- 
gression from mild cognitive impairment to dementia. Dementia y Neuropsychologia, 4(1), 28-34. https://doi.org/10.1590/ S1980-57642010DN40100005

Asociación Americana de Terapia Ocupacional [AOTA] (2008). Occupational therapy practice framework: Domain and process (2nd ed.). American Journal of Occupational Therapy, 62, 625-683. https://doi. org/10.5014/ajot.62.6.625

Antonovsky, A. y Sagy, S. (1990). Confronting developmental tasks in the retirement transition. Gerontologist, 30, 362-368. https://doi.org/10.1093/geront/30.3.362

Arenaza, M. y Bartréz, D. (2014). Reserva cognitiva. En D. Redolar (Ed.), Neurociencia cognitiva (pp. 185-197). Editorial Médica Panamericana.

Baltes, P. B., Lindenberger, U. y Staudinger, U. M. (2006). Life-span theory in developmental psychology. En W. Damon y R. M. Lerner (Eds.), Handbook of child psychology: Theoretical models of human development (pp. 569-664). Wiley.

Berger, S. (2009). Psicología del desarrollo. Adultez y vejez. Editorial Médica Panamericana.

Bruner, J. (1990). Actos de significado. Alianza.

Burton, J. E. (1989). The Model of Human Occupation and occupational therapy practice with elderly patients. Part 1: Characteristics of ageing. British Journal of Occupational Therapy, 52(6), 215-218. https:/doi. org/ 10.1177/030802268905200603

Cabral, Y.P.S., Carvalho, C.R.A. y Gonçalves, M.V. (2018). Publicações brasileiras de terapia ocupacional na área de gerontologia entre 2010 e 2015: uma revisão de escopo. Revista Ocupa- ción Humana, 18 (2), 20-40. https://doi. org/10.25214/25907816.226

Calasanti, T. y King, N. (2015). Intersectionality and age. En J. Twigg y W. Martin (Eds.), Routledge handbook of cultural gerontology (pp.193-200). Routledge.

Carvalho, C. R. A. (2018). A inserção dos terapeutas ocupacionais nos diferentes contextos de atenção à pessoa idosa. En L. D. Bernardo \& T. M. Raymundo (Ed.), Terapia Ocupacional e Gerontologia: Interlocuções e Práticas (pp.127-140). Appris.

Carvalho, C., Malfitano, A.P.S. y Lopes, R.E. (2018). Intervenciones sociales de terapia ocupacional con adultos mayores en América del Sur: una revisión no sistemática de la literatura. Revista Chilena de Terapia Ocupacional, 18(2), 29 - 43.

Corregidor, A. (2010). Terapia ocupacional en geriatría y gerontología. Bases conceptuales y aplicaciones prácticas. Ergon.

Cruz, A., Arias, M., Figueroa, C., LLavona, D. y Rivera, A. (2014). Factores que promueven la adaptación ocupacional de adultos mayores en Puerto Rico. TOG (A Coruña), 11(20), 21. http://www.revistatog. com/num20pdfs/original10.pdf

Da Rocha Medeiros, M. (2008). Terapia ocupacional. Un abordaje epistemológico y social. Universidad Nacional del Litoral.

De las Heras, C. (2015). Modelo de Ocupación Humana. Síntesis.

Dulcey, E. (2010). Psicología social del envejecimiento y perspectiva del transcurso de la vida. Revista Colombiana de Psicología, 19(2), 207-224. http://www.bdigital.unal. edu.co/18647/2/14521-131949-1-PB.htm 
Duque, J. (2010). Perspectiva ocupacional de las personas mayores. En A. Corregidor (coord.), Terapia ocupacional en geriatría y gerontología (pp. 19-26). Sociedad Española de Geriatría y Gerontología.

Elliott, M. S. y Barris, R. (1987). Occupational role performance and life satisfaction in elderly persons. The Occupational Therapy Journal of Research, 7(4), 215-224. https:/ doi.org/10.1177/153944928700700403

Fallahpour, M., Borell, L., Luborsky, M. y Nygard, L. (2015). Leisure-activity participation to prevent later-life cognitive decline: a systematic review. Scandinavian Journal of Occupational Therapy, 23(3),162197. https:/doi.org/10.3109/11038128.20 15.1102320

Fernández, R. (2000). La gerontología positiva. Revista Multidisciplinar de Gerontología, 10(3), 143-145.

Fernández, R. (2007). Envejecimiento saludable: Aspectos biológicos, Psicológicos y sociales. Universitas-UNED.

Fernández, R., Zamarrón, M. D., López, M., Molina, M. A., Diez, J., Montero, P. y Schettini, R. (2010). Envejecimiento con éxito: criterios y predictores. Psicothema, 22(4), 641-647. http://hdl.handle. net/10261/83676

Ferraro K. y George, L. (2016). Handbook of aging and the social sciences - 8th Edition. Academic Press.

Francisco, B. R. (1988). Terapia Ocupacional. Papirus.

Galheigo, S. M. (2012). Perspectiva crítica y compleja de terapia ocupacional: actividad, cotidiano, diversidad, justicia social y compromiso ético-político. TOG
(A Coruña), 5, 176-187. http://www.revistatog.com/mono/num5/compromiso.pdf

Gergen, K. J. y Gergen, M. M. (1988). Narrative and the self as relationship. En L. Berkowitz (Ed.), Advances in experimental social psychology (pp. 17-56). Academic.

Guajardo, A. (2012). Enfoque y praxis en terapia ocupacional: reflexiones desde una perspectiva de la terapia ocupacional crítica. TOG (A Coruña), 5, 18-29. http://www.revistatog.com/mono/num5/ prologo.pdf

Gubrium, J. F. (1993). Contributions of qualitative research on aging. Qualitative Health Research, 3(2), 131-132. https://doi. org/10.1177/104973239300300201

Harrris, P. y Allegri, R. (2009). Reserva cognitiva y su efecto protector frente a la patología cerebral. Archivos de Neurología, Neurocirugía y Neuropsiquiatría, 18, 38-48.

Horstead, K. y Bluestone, K. (2018). Who cares? Why older women's economic empowerment matters for the Sustainable Development Goals. Age International. https://www.ageinternational. org.uk/contentassets/b73c56b5662f44c2988f4018f1acb679/who-cares---age-international---nov18.pdf

Hwang, J. E. (2012). Development and validation of a 15-item lifestyle screening for community-dwelling older adults. American Journal of Occupational Therapy, 66, e98-e106. https:/ doi.org/10.5014/ajot.2012.005181

Leal, E. El derecho a espacios óptimos para todos. Enlace En Red, 31, 33-35. https://www. imserso.es/InterPresent1/groups/imserso/documents/binario/enlace31 completa.pdf 
Jackson, J., Clarson, M., Mandel, D., Zemke, R. y Clark, F. (1998). Occupation in lifestyle redesign: The well elderly study occupational therapy program. The American Journal of Occupational Therapy, 2(5), 326-336. https:/doi. org/10.5014/ajot.52.5.326

Johansson, A. y Björklund, A. (2016). The impact of occupational therapy and lifestyle interventions on older persons' health, well-being, and occupational adaptation: A mixed-design study. Scandinavian Journal of Occupational Therapy, 23(3), 207-219. http://doi.org/10.3 109/11038128.2015.1093544

Jones, R., Manly, J., Glymour, M. y Rentz, D. (2014). Conceptual and measurement challenges in research on cognitive reserve. Journal of the International Neuropsychological Society, 17(4), 593-601. https://doi.org/10.1017/ $\underline{\mathrm{S} 1355617710001748}$

Jonsson, H., Josephsson, S. y Kielhofner, G. (2000). Evolving narratives in the course of retirement: A longitudinal study. American Journal of Occupational Therapy, 54, 463-470. https://doi.org/10.5014/ ajot.54.5.463

Jonsson, H., Josephsson, S. y Kielhofner, G. (2001). Narratives and experience in an occupational transition: A longitudinal study of the retirement process. American Journal of Occupational Therapy, 55, 424432. https://doi.org/10.5014/ajot.55.4.424

Jonsson, H., Kielhofner, G. y Borell, L. (1997). Anticipating retirement: The formation of narratives concerning an occupational transition. American Journal of Occupational Therapy, 51, 49-56. https://doi. org/10.5014/ajot.51.1.49
Kielhofner, G. (2004). Modelo de Ocupación Humana ( $3^{\mathrm{a}}$ ed.). Editorial Médica Panamericana.

Kielhofner, G. (2011). Modelo de Ocupación Humana. Teoría y Aplicación ( $4^{\mathrm{a}}$ ed). Editorial Médica Panamericana.

Kronenberg, F., Simó, S. y Pollard N. (2005). Occupational therapy without borders: learning from the spirit of survivors. Elsevier.

Krzemien, D. (2006). Afrontamiento a situaciones de crisis en la vejez femenina. Anales de Psiquiatría, 22(4), 171.

Krzemien, D. (2013). El campo multidisciplinario de la gerontología. Debate actual sobre demografía, desarrollo social e investigación del envejecimiento. Académica Española.

Lalive d'Epinay, Ch., Bickel, J. F., Cavalli, S. y Spini, D. (2005). Le parcours de vie: émergence d'un paradigme interdisciplinaire. En J. F. Guillaume (Ed.), Parcours biographiques (pp.187-210). De Boeck.

Liberalesso, A. (2007). El legado de Paul B. Baltes a la psicología: el paradigma Life Span aplicado al desarrollo y al envejecimiento. Revista Latinoamericana de Psicología, 39(2), 413-417.

Lombardo, E. y Krzemien, D. (2008). La Psicología del curso de vida en el marco de la psicología del desarrollo. Revista Argentina de Sociología, 6(10), 111- 120. http://www. scielo.org.ar/pdf/ras/v6n10/v6n10a08.pdf

Miranda, C. (2005). La ocupación en la vejez. Una visión gerontológica desde terapia ocupacional. Ediciones Suárez. 
Morrison, R. (2018). O que une a Terapia Ocupacional? Paradigmas e perspectivas ontológicas da ocupação humana. Revisbrato, 2(1), 182-203. https://revistas.ufrj.br/index.php/ribto/article/view/12699

Morrison, R., Guajardo, A. y Schliebener, M. (2016). Conferencia: Debates y reflexiones para una ciencia de la ocupación crítica y social. Diálogos para comprender la ocupación humana. Revista Argentina de Terapia Ocupacional, 1(2), 40-58. http:// www.revista.terapia-ocupacional.org.ar/ RATO/2016jul-conf.pdf

Mulholland, F. y Jackson, J. (2018). The experience of older adults with anxiety and depression living in the community: Aging, occupation and mental wellbeing. British Journal of Occupational Therapy, 1-10. https:/doi.org/ $\underline{10.1177 / 0308022618777200}$

Oakley, F. (2008). Clinical application of the Model of Human Occupation in dementia of the Alzheimer's type. Journal Occupational Therapy in Mental Health, 37-50. https://doi.org/10.1300//004v07n04 04

Organización Iberoamericana de Seguridad Social [OISS] (2016). Boletín periódico del programa iberoamericano de cooperación sobre adultos mayores No. 18. OISS. https://oiss.org/ wp-content/uploads/2000/01/BOLETIN 18-ODS-1707def.pdf

Organización Mundial de la Salud [OMS] (2015). Informe mundial sobre el envejecimiento y la salud (NLM: WT 104). OMS. https://www.who.int/ageing/publications/world-report-2015/es/
Palma, O., Hueso, C., Martí, C., Fernández, M., Campos, C. P. y Montoya, R. (2019). Understanding the occupational adaptation process and well-being of older adults in Magallanes (Chile): A qualitative study. International Journal of Environmental Research and Public Health, 16(19), 36-40. http://doi.org/10.3390/ijerph16193640

Perlmutter, M.S., Bhorade, A., Gordon, M., HoIlingsworth, H. H. y Baum, M. C. (2010). Cognitive, visual, auditory, and emotional factors that affect participation in older adults. American Journal of Occupational Therapy, 64(4), 570-579. https:/doi. org/10.5014/ajot.2010.09089

Peterson, E., Howland, J., Kielhofner, G., Lachman, M. E., Assmann, S., Cote, J. y Jette, A. (1999). Falls self-efficacy and occupational adaptation among elders. Physical and Occupational Therapy, 38, 257. https://doi.org/10.1080//148v16n01 01

Pozo, M. (2017). La red mundial de ciudades y comunidades amigables con las personas mayores. Enlace En Red, 31, 25-30. https://www.imserso.es/InterPresent1/ groups/imserso/documents/binario/enlace31completa.pdf

Redolar, R. (2014). Neurociencia cognitiva. Editorial Médica Panamericana.

Rowe, J. W. y Kahn, R. L. (1987). Human aging: usual and successful. Science, 237(4811), 143-149. http://doi.org/10.1126/science.3299702

Rubinstein, R. L. y de Medeiros, K. (2015). "Successful aging", gerontological theory and neoliberalism: A qualitative critique. The Gerontologist, 55(1), 34-42. https:// doi.org/10.1093/geront/gnu080 
Rudman, D. L. (2015). Embodying positive aging and neoliberal rationality: Talking about the aging body within narratives of retirement. Journal of Aging Studies, 34, 10-20. https://doi.org/10.1016/j.jaging.2015.03.005

Sanders, M. J. (2018). Older manufacturing workers and adaptation to age-related changes. American Journal of Occupational Therapy, 72, 1-11. https:/doi. org/10.5014/ajot.2018.021238

Schon, D. (1979). Generative metaphor: A perspective on problem-setting in social policy. En A. Ortony (Ed.), Metaphor and thought (pp.137-163). Cambridge University Press. https://doi.org/10.1017/ CBO9781139173865.011

Shotter, J. (2001). Realidades conversacionales. Amorrortu.

Simó, S., Guajardo, A., Corrêa, F., Galheigo, S. y García, S. (2016). Terapias Ocupacionales desde el Sur Derechos humanos, ciudadanía y participación. Editorial Universidad de Santiago de Chile.

Smallfield, S. y Molitor, W. L. (2018). Occupational therapy interventions supporting social participation and leisure engagement for community-dwelling older adults: A systematic review. American Journal of Occupational Therapy, 72(4), 1-8. https:// doi.org/10.5014/ajot.2018.030627

Soto, M. y Arcos, M. (2010). Reserva cognitiva y rendimiento neuropsicológico en una muestra de adultos mayores de la ciudad de Arequipa. Revista de Investigación Universidad Católica San Pablo, 1, 6-2.

Stern, Y. (2011). Elaborating a hypothetical concept: Comments on the special series on cognitive reserve. Journal of the International Neuropsychological Society,
17(4), 639-642. https://doi.org/10.1017/ $\underline{\text { S1355617711000579 }}$

Taylor, R.R. y Kielhofner, G. (2017). Kielhofner's model of human occupation: theory and application. Wolters Kluwer.

Trentham, B. L. y Neysmith, S. M. (2018). Exercising senior citizenship in an ageist society through participatory action research: A critical occupational perspective. Journal of Occupational Science, 25(2), 174190. https://doi.org/10.1080/14427591.2 $\underline{017.1402809}$

Turcotte, P. L., Carrier, A., Roy, V. y Levasseur, M. (2018). Occupational therapists' contributions to fostering older adults' social participation: A scoping review. British journal of occupational therapy, 81(8), 427-449. https://doi. org/10.1177/0308022617752067

Vigotsky, L. S. (1979). El desarrollo de los procesos psicológicos superiores. Grijalbo.

Villar, F. (2005). El enfoque del ciclo vital: hacia un abordaje evolutivo del envejecimiento. En S. Pinazo Hernandis y M. Sánchez Martínez (Dir.) Gerontología: actualización, innovación y propuestas (pp. 148-181). Pearson.

Yarce, E., Rosas, G., Paredes, Y., Rosero, M. y Morales, A. (2014). Intereses ocupacionales de adultos mayores de 60 años de la ciudad San Juan de Pasto. Revista UNIMAR, 33(1), 201-212.

Yerxa, E. (1990). An introduction to occupational science. A foundation for occupational therapy in the 21st century. Occupational Therapy in Health Care, 6(4), 1-17. https://doi.org/10.1080//003v06n04_04 
Yerxa, E. (1998). Health and the human spirit for occupation. American Journal of Occupational Therapy, 52, 412-418. https:/ doi.org/10.5014/ajot.52.6.412

Yuni, J. y Urbano, C. (2016). Envejecer aprendiendo. Claves para un envejecimiento activo. Editorial Brujas. 